\title{
Autophagy as a target for cancer therapy: new developments
}

\author{
This article was published in the following Dove Press journal: \\ Cancer Management and Research \\ 10 October 2012 \\ Number of times this article has been viewed
}

\section{Jennifer S Carew \\ Kevin R Kelly \\ Steffan T Nawrocki}

The Department of Medicine and Institute for Drug Development, Cancer Therapy and Research Center at The University of Texas Health Science Center, San Antonio, TX, USA
Correspondence: Steffan T Nawrocki Department of Medicine and Institute for Drug Development, Cancer Therapy and Research Center at The University of Texas Health Science Center, 7979 Wurzbach Road, San Antonio, TX 78229, USA

$\mathrm{Tel}+\mathrm{I} 2104503894$

Fax + I 21045084 II

Email nawrocki@uthscsa.edu
Abstract: Autophagy is an evolutionarily conserved lysosomal degradation pathway that eliminates cytosolic proteins, macromolecules, organelles, and protein aggregates. Activation of autophagy may function as a tumor suppressor by degrading defective organelles and other cellular components. However, this pathway may also be exploited by cancer cells to generate nutrients and energy during periods of starvation, hypoxia, and stress induced by chemotherapy. Therefore, induction of autophagy has emerged as a drug resistance mechanism that promotes cancer cell survival via self-digestion. Numerous preclinical studies have demonstrated that inhibition of autophagy enhances the activity of a broad array of anticancer agents. Thus, targeting autophagy may be a global anticancer strategy that may improve the efficacy of many standard of care agents. These results have led to multiple clinical trials to evaluate autophagy inhibition in combination with conventional chemotherapy. In this review, we summarize the anticancer agents that have been reported to modulate autophagy and discuss new developments in autophagy inhibition as an anticancer strategy.

Keywords: autophagy, chloroquine, lucanthone, cancer, apoptosis

\section{Introduction}

Autophagy is an evolutionarily conserved cellular catabolic degradation process that is characterized by the formation of double-membrane vesicles (autophagosomes) that engulf cellular components targeted for destruction. Autophagic degradation is an important regulator of cellular homeostasis as this process mediates the turnover of defective organelles, misfolded or aggregated proteins, and certain longlived molecules. ${ }^{1}$ Knockout animal studies demonstrated that some degree of basal autophagy is essential for viability as autophagy-deficient mice cannot survive the neonatal starvation period, and these mice die within one day of birth. ${ }^{2}$ Ex vivo studies demonstrating that autophagy-deficient cells are significantly more susceptible to starvation-mediated cell death triggered by serum or growth factor deprivation than their autophagy-competent counterparts provided further support for a critical role for autophagy in the regulation of cellular homeostasis. ${ }^{3}$ Autophagy is initiated by stress signals from the mammalian target of rapamycin complex 1 (mTORC1), which activates the kinase ULK1 (ATG1), and which then forms a complex with ATG13 and ATG17. ${ }^{4}$ Autophagosome formation occurs after mTORC1 activity is inhibited and requires class 3 phosphoinositide 3-kinase (PI3K) activity as vacuolar sorting protein 34 forms a complex with Beclin-1. ${ }^{5}$ Microtubule-associated protein light chain 3 (LC3) is incorporated into the membrane by ATG7 and ATG3. LC3 recruits adaptor proteins such as p62 and NIX that recognize protein aggregates and damaged organelles and 
recruits them into the autophagosome. ${ }^{6,7}$ Autophagosomes subsequently fuse with lysosomes to form autolysosomes, and their cargo is degraded by a number of different lysosomal proteases including the cathepsins (Figure 1). In this review, we will summarize what is currently known regarding the regulation of autophagy, the role(s) of autophagy within the context of malignancy, and the therapeutic implications of autophagy activation versus autophagy inhibition in the treatment of cancer.

\section{Established regulators of autophagy Mammalian Target of Rapamycin (mTOR)}

Although a number of signaling pathways have been implicated in the control of autophagy, the most well characterized autophagy regulator to date is mTOR. mTOR functions as two multiprotein complexes, mTORC1 and mTORC2, and each has unique binding partners and differential sensitivity to rapamycin and related compounds. ${ }^{8}$ mTORC 1 is able to form a complex with multiple binding partners, and its activity is inhibited by rapamycin and related drugs including temsirolimus/CCI-779, everolimus/RAD001, and ridaforolimus/AP23573. ${ }^{9-13}$ The PI3K/AKT/mTOR signaling cascade is an essential regulator of protein translation and cell proliferation. Its activity can be stimulated by growth factors and nutrients, although this pathway is constitutively active in many cancer types.

Constitutive PI3K/AKT signaling in malignant cells is frequently a consequence of mutations in PI3K or upstream growth factor receptors, AKT overexpression, or it can

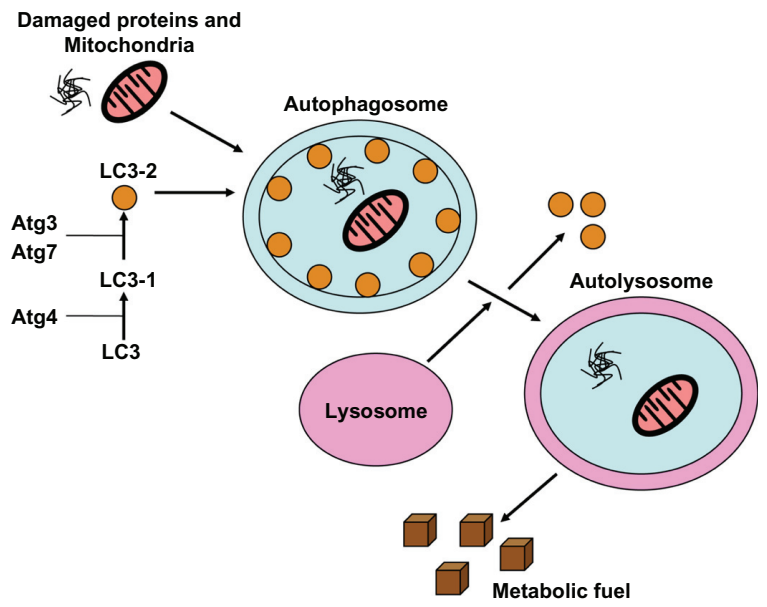

Figure I Autophagy produces metabolic fuel through the degradation of biomolecules. Notes: Damaged proteins, organelles, and other biomolecules are sequestered into double-membrane vesicles called autophagosomes. LC3 is essential for autophagosome maturation. The mature autophagosomes fuse with the lysosome, and biomolecules are degraded by hydrolytic enzymes into metabolic fuel. Abbreviation: LC3, lipidated cytosolic-associated protein light chain. occur due to loss of the tumor suppressor phosphatase and tensin homolog (PTEN) on chromosome ten, which functions as a negative regulator of this pathway. ${ }^{14,15}$ Since activation of the PI3K/AKT cascade promotes mTOR activity, many tumor types exhibit high levels of mTOR activity due to constitutive upstream signaling events. In addition to its regulation by $\mathrm{PI} 3 \mathrm{~K} / \mathrm{AKT}$, $\mathrm{mTOR}$ activity is also controlled by AMP-activated kinase (AMPK), which functions as a sensor for cellular nutrient and energy levels. Upon its activation, mTORC1 stimulates protein synthesis via phosphorylation of the elongation factor 4E-BP1 and p70 S6 kinase. When nutrient and energy supplies are adequate, mTORC1 suppresses autophagy through the phosphorylation of the autophagy kinases ULK1 and ULK2. ${ }^{16-18}$

There are a number of different mechanisms through which the autophagy-related functions of the PI3K/AKT/ mTOR pathway are regulated. For example, mTORC2 can inhibit autophagy through the phosphorylation of AKT. ${ }^{19}$ Conversely, when levels of nutrients and metabolic fuel are diminished, the energy sensor AMPK can be activated upon its phosphorylation by the serine-threonine kinase LKB1. This subsequently leads to the activation of tuberous sclerosis protein (TSC2), a consequential reduction in mTORC1 activity, and the stimulation of autophagy. The activation of autophagy serves to maintain cell survival under nutrient-deficient conditions, as the breakdown of cellular components generates essential metabolic fuel. Similarly, increased autophagic activity has been frequently observed in malignant cells in response to treatment with therapeutic mTOR inhibitors, and this has been hypothesized to significantly reduce their clinical activity. ${ }^{20}$

\section{Beclin- I}

Beclin-1 is a member of the BCL-2 homology domain 3 (BH3) containing proteins, which have been well characterized as critical regulators of apoptosis. ${ }^{21}$ Beclin-1 is widely expressed in human tissues and is primarily localized to the endoplasmic reticulum, mitochondria, and the perinuclear membrane. Earlier studies demonstrated that the evolutionarily conserved domain of Beclin-1 is required for its proautophagic and tumor suppressive functions. The BH3 domain of Beclin-1 facilitates its selfassociation and its interactions with antiapoptotic members of the BCL-2 family. Recent studies have identified death-associated protein kinase (DAPK) as an important regulator of Beclin-1-mediated autophagy. ${ }^{22}$ Phosphorylation of Beclin-1 by DAPK causes it to dissociate from BCL-2 family members and this event promotes the 
induction of autophagy. ${ }^{22}$ Interestingly, DAPK has also been shown to have a Beclin-1 independent function in the stimulation of autophagy through its interactions with LC3.

A separate investigation defined an indirect role for c-Jun N-terminal kinase 1 (JNK1) in the control of Beclin-1-associated autophagy by demonstrating that JNK-mediated phosphorylation of BCL-2 disrupts its binding to Beclin-1, which ultimately leads to the induction of autophagy. ${ }^{23}$ Ubiquitination also appears to contribute to the control of Beclin-1 function as its ubiquitination on lysine 63 was recently shown to facilitate its self-association and the formation of autophagosomes. ${ }^{24}$ Additional studies are required to fully elucidate the finer mechanistic details regarding how different phosphorylation and ubiquitination events specifically control Beclin-1's function within the context of autophagy. Finally, the generation of Beclin-1-deficient mice established the essential function of Beclin-1-mediated autophagy during embryonic development, as its loss yields an embryonic lethal phenotype characterized by death at embryonic day 7.5 or earlier due to impaired proamniotic canal closure. ${ }^{25,26}$

\section{p53}

The identification of the p53 transcriptional target damageregulated autophagy modulator (DRAM) established a role for p53 in the regulation of autophagy. Initial studies demonstrated that DRAM1 expression was induced by DNAdamaging agents in a p53-dependent manner. ${ }^{27,28}$ Indications that DRAM1 may play a role in autophagy were initially revealed by its lysosomal localization. Mechanistic studies designed to elucidate DRAM1's function(s) demonstrated that its expression promotes autophagosome formation. ${ }^{27,28}$ A subsequent study showed that DRAM could also be activated by the $\mathrm{p} 53$ related protein, $\mathrm{p} 73$. However, unlike p53, p73 retained its ability to induce autophagy in a manner that was independent of DRAM1 expression. ${ }^{29}$ These findings indicated that other $\mathrm{p} 53 / \mathrm{p} 73$ transcriptional targets may be able to compensate for the loss of DRAM1 with respect to the regulation of autophagy.

Several studies have identified a number of candidate transcriptional targets that may contribute to the control and execution of autophagy. ${ }^{22,30-32}$ Additional research is required to define their individual roles and significance. Interestingly, more recent rigorous analyses of DRAM1 and its functions showed that DRAM1 encodes multiple isoforms and also belongs to a family of five related proteins, whose functions and transcriptional regulation remain to be fully elucidated. ${ }^{33,34}$ Ongoing studies will likely clarify these issues.

\section{Autophagy in cancer}

The roles of autophagy within the context of cancer remain somewhat controversial and appear to be quite divergent in the pre- and postmalignant states. Numerous studies have established that autophagy is an essential process in tumor suppression. The first evidence of this came from a study of mice with Beclin-1 haploinsufficiency $\left(\mathrm{BECN1}^{+/-}\right)$, which displayed a significantly higher frequency of spontaneous malignancies (leukemias, lymphomas, and tumors of the lung and liver) as compared with their wild-type counterparts. ${ }^{26} \mathrm{As}$ mentioned earlier, deletion of both Beclin-1 alleles induces embryonic lethality. ${ }^{25,26}$ These phenotypic characteristics most likely result from the aberrant accumulation of organelles and specific proteins such as ubiquitinated keratins and the autophagy cargo adaptor p62 due to compromised autophagic degradation. ${ }^{35}$ In particular, abrogation of p62 turnover due to the disruption of autophagy has been shown to lead to activation of the DNA damage response, disruption of cellular redox status, accumulation of defective mitochondria, increased susceptibility to degenerative and inflammatory diseases, and enhanced tumorigenicity. ${ }^{35-38}$ Specifically, several clinical correlative studies have provided additional support for a role for Beclin-1 in tumor suppression. The loss of Beclin-1 expression is linked to a poor prognosis in patients with solid malignancies. Low/deficient Beclin-1 expression is associated with shorter overall survival in patients with colon cancer and squamous-cell carcinoma of the esophagus. ${ }^{39,40}$ Conversely, elevated Beclin-1 levels appear to be associated with a favorable prognosis in patients with high-grade glioblastomas or hepatocellular tumors. ${ }^{41,42}$

However, a separate study of specimens from patients with nasopharyngeal tumors showed that high Beclin-1 expression was significantly correlated with poor outcomes. ${ }^{43}$ Similarly, two independent studies investigated LC3 expression levels and their potential correlation with clinical outcomes in patients with cancer. A study of melanoma in situ (MIS) and melanoma specimens showed significantly higher levels of expression of LC3 and greater numbers of autophagosomes in both tissue types as compared with normal melanocytes and early stage melanoma in situ, indicating that the elevation of autophagic activity may be a later event in disease pathogenesis. ${ }^{44} \mathrm{~A}$ larger assessment of LC3 levels in gastrointestinal tumors demonstrated overexpression of LC3 in more than 50\% of all specimens analyzed. Interestingly, there was no clear link between LC3 levels and patient survival. ${ }^{45}$ Collectively, these findings outline a potential role for autophagy as a contributor to the malignant phenotype, but suggest that caution should be taken regarding 
the potential use of any individual autophagy factor as a predictive biomarker.

The majority of pertinent studies conducted to date indicate that autophagy likely contributes to tumor suppression in healthy cells. However, it appears that autophagy may instead promote the pathogenesis of established malignancies by providing cancer cells with a mechanism to generate alternative sources of metabolic fuel via protein recycling, which facilitates their survival in nutrient/oxygen-deficient conditions. ${ }^{46,47}$ Considering that nutrient and oxygen deprivation are hallmark features of the tumor microenvironment, cancer cells may have an increased dependency upon autophagy for their survival. A number of studies have provided support for this hypothesis. For example, hypoxia-inducible transcription factor $1 \alpha$ (HIF-1 $\alpha$ ), which is constitutively active in many tumor types, was shown to promote BCL-2/adenovirus E1B 19kDa-interacting protein (BNIP3L)-dependent autophagy. ${ }^{48,49}$ Recent evidence suggests that hypoxic conditions may also trigger autophagy through a HIF-1 $\alpha$-independent mechanism that involves activation of the unfolded protein response. ${ }^{50}$ Unfolded protein response-induced autophagy may serve as a mechanism to reduce endoplasmic reticular energy consumption while simultaneously generating metabolic fuel from the catabolism of endoplasmic reticulum membranes and other organelles such as mitochondria. ${ }^{51}$

While most published studies support a prosurvival role for autophagy in cancer pathogenesis, there is also evidence for the induction of autophagy during the cell death process. In fact, some investigators in the field believe that autophagy can function as a unique caspase-independent mechanism of cell death that is distinct from apoptosis and necrosis as the minimum number of organelles or cellular components are degraded as required to support cell survival. ${ }^{52}$ Others feel that autophagy associated with cell death likely reflects an attempt by cancer cells to survive the stress stimuli that initiated their death process and that it impedes rather than accelerates the efficiency of apoptotic/necrotic execution. The specific implications of this phenomenon are likely to be context-dependent and vary between in vitro and in vivo settings. Additional research is required in order to draw definitive conclusions. These reviews provide an excellent summary of what is currently known regarding autophagy as a potential mechanism of cell death. ${ }^{53,54}$

A number of studies have provided strong evidence implicating autophagy as a process that increases the survival capacity of both normal and malignant cells under stressful and metabolically challenging conditions both in culture and in the in vivo microenvironment. ${ }^{2,47,55}$ Genetic impairment of autophagy significantly diminishes the ability of cells to withstand nutrient deprivation and confers an accelerated rate of cell death in response to these conditions as compared with experimental controls. ${ }^{38}$ Although most evidence suggests that the survival advantage of autophagy-competent versus autophagy-deficient cells in the face of oxidative, genotoxic, or metabolic stress is derived from the autophagy-mediated catabolic generation of alternative energy sources, it is possible that other aspects of autophagy function could also promote cell survival and/or resistance to cell death. Ongoing research will likely define additional roles for autophagy in the regulation of cell survival.

Although low nutrient levels and hypoxia have been shown to trigger autophagy in cancer cells, numerous studies have shown that autophagy may also be stimulated in response to treatment with anticancer agents and radiation therapy. A wide array of therapeutic modalities with diverse mechanisms of action have been reported to induce autophagy including mTOR inhibitors, arsenic trioxide, etoposide, histone deacetylase (HDAC) inhibitors, tamoxifen, temozolomide, tyrosine kinase inhibitors, proteasome inhibitors, and ionizing radiation (Table 1). ${ }^{56-62}$ Based on earlier studies that showed that autophagy can represent both an alternative cell death pathway and a mechanism that promotes survival, autophagy is frequently viewed as a "double-edged sword."

The role that autophagy plays following chemotherapy remains highly controversial. It is possible that the induction of autophagy may contribute to the efficacy of some anticancer agents; conversely, it could also facilitate cell survival by maintaining bioenergetics following exposure to chemotherapeutic agents. ${ }^{1}$ In order to better define the role of autophagy in the regulation of sensitivity to cancer therapeutics, investigators have quantified the impact of autophagy inhibition on the efficacy of numerous anticancer agents, including vorinostat, cyclophosphamide, and imatinib. ${ }^{56,57,59,60,63}$ The overwhelming majority of relevant preclinical studies have demonstrated that disruption of autophagy significantly augments the efficacy of most classes of anticancer agents. ${ }^{56-59,63}$ These studies suggest that autophagy inhibition may be an effective approach worthy of further investigation with broad applications in cancer therapy.

\section{Therapeutic modulation of autophagy: new developments}

As mentioned earlier, inhibition of autophagy in preclinical models increases the sensitivity of tumors to diverse 
Table I Selected agents that modulate autophagy

\begin{tabular}{|c|c|c|c|}
\hline Drug class & Agent & Target & Status \\
\hline \multicolumn{4}{|l|}{ Autophagy inducers } \\
\hline \multirow[t]{3}{*}{ mTOR inhibitors } & Temsirolimus $^{70}$ & mTOR & Approved (RCC) \\
\hline & Sirolimus $^{71}$ & & Approved (Kidney transplants) \\
\hline & Everolimus $^{72}$ & & Approved (RCC) \\
\hline \multirow[t]{2}{*}{ HDAC inhibitors } & Vorinostat ${ }^{73}$ & HDACs & Approved (CTCL) \\
\hline & Panobinostat ${ }^{74}$ & & Phase 3 \\
\hline \multirow[t]{2}{*}{ Proteasome inhibitors } & Bortezomib $^{75}$ & Proteasome & Approved (MM, MCL) \\
\hline & NPI-0052 $2^{62}$ & & Phase I \\
\hline \multirow[t]{4}{*}{ Tyrosine kinase inhibitors } & Imatinib ${ }^{76-78}$ & BCR-ABL, KIT, PDGFR & Approved (CML, GIST) \\
\hline & Dasatinib $^{79}$ & BCR-ABL, SRC & Approved (CML) \\
\hline & Sorafenib ${ }^{80}$ & Multi (VEGFR, RAF, KIT) & Approved (RCC, HCC) \\
\hline & Lapatinib ${ }^{81}$ & EGFR, HER2 & Approved (Breast cancer) \\
\hline Estrogen receptor antagonist & Tamoxifen $^{82}$ & Estrogen receptor & Approved (Breast cancer) \\
\hline \multirow[t]{3}{*}{ Antibodies } & Rituximab $^{83}$ & CD20 & Approved (Lymphoma) \\
\hline & Panitumumab ${ }^{84}$ & EGFR & Approved (Colon cancer) \\
\hline & Cetuximab $^{85}$ & EGFR & Approved (Colon and head/neck cancer) \\
\hline Alkylating agents & Temozolomide $^{86}$ & DNA & Approved (Glioblastoma) \\
\hline \multirow[t]{2}{*}{$\mathrm{BH} 3$ mimetics } & GXI5-07080 & $\mathrm{Bcl}-2$ & Phase 2 \\
\hline & $\mathrm{ABT} 737^{87}$ & & Preclinical \\
\hline \multicolumn{4}{|l|}{ Autophagy inhibitors } \\
\hline \multirow[t]{3}{*}{ Aminoquinolines } & Chloroquin $e^{56,59,63}$ & Lysosome & Approved (Malaria) \\
\hline & Lys $05^{69}$ & & Preclinical \\
\hline & Hydroxychloroquine $e^{56}$ & & $\begin{array}{l}\text { Approved (Malaria, systemic lupus erythematosus, } \\
\text { rheumatoid arthritis) }\end{array}$ \\
\hline Thioxanthenones & Lucanthone ${ }^{58}$ & $\begin{array}{l}\text { Lysosome, topoisomerase } 2 \text {, } \\
\text { AP endonuclease }\end{array}$ & Approved (Schistosomiasis) \\
\hline
\end{tabular}

Abbreviations: mTOR, mammalian target of rapamycin; RCC, renal cell carcinoma; HDAC, histone deacetylases; CTCL, cutaneous T-cell lymphoma; MM, multiple myeloma; MCL, mantle cell lymphoma; PDGFR, platelet-derived growth factor receptor; CML, chronic myelogenous leukemia; GIST, gastrointestinal stromal tumor; VEGFR, vascular endothelial growth factor receptor; HCC, hepatocellular carcinoma; EGFR, epidermal growth factor receptor.

classes of anticancer agents. ${ }^{64}$ Chloroquine (CQ) and its analog, hydroxychloroquine (HCQ), are the only autophagic inhibitors currently being evaluated in clinical trials for cancer therapy. Both of these drugs disrupt lysosomal function and prevent the degradation of proteins within the autophagosome. HCQ is approved by the Food and Drug Administration for the treatment of malaria, rheumatoid arthritis, and lupus, and is currently being evaluated in numerous cancer clinical trials. ${ }^{65}$ Most of these clinical studies are investigating HCQ in combination with standard of care agents (Table 2). Although these agents hold promise for their potential applications in cancer therapy due to their ability to inhibit autophagy and increase the efficacy of many standard of care treatments, CQ and HCQ induce ocular toxicities, such as retinopathy. It is also still unclear whether safely tolerated doses of HCQ or CQ can effectively and consistently inhibit autophagy in human tumors. These issues underscore the need for additional inhibitors of autophagy.

Recent preclinical studies have identified two new autophagy inhibitors with potential clinical utility in cancer therapy, and presented with some advantages associated with CQ/HCQ. The first agent, lucanthone (Miracil D), is an existing drug that has been used for many years for the treatment of schistosomal parasites. Earlier investigations have shown that lucanthone inhibits topoisomerase 2 activity, as well as the activity of AP endonuclease, a critical regulator of DNA base excision repair. ${ }^{66,67}$ These findings prompted the evaluation of lucanthone as a potential sensitizer to chemotherapy and radiation. A more recent study defined a novel mechanism of action for lucanthone that includes the disruption of lysosomal function, inhibition of autophagy, and induction of apoptosis. ${ }^{58}$ Lucanthone triggered apoptosis in cancer cells independently of p53 functional status. Moreover, knockdown studies demonstrated that the lysosomal protease cathepsin D was an important regulator of the proapoptotic effects of lucanthone. Notably, a direct comparison of the cellular sensitivity of a panel of human breast cancer cells to lucanthone and CQ showed that lucanthone was approximately tenfold more potent than CQ in this setting. The fact that earlier clinical studies of lucanthone did not demonstrate any evidence of drug-related ocular toxicity suggests that in addition to possessing increased anticancer activity, lucanthone may also have a better safety profile than $\mathrm{CQ} / \mathrm{HCQ}{ }^{68}$ These collective findings provide a strong rationale for the 
Table 2 Selected clinical trials with the autophagy inhibitor HCQ

\begin{tabular}{|c|c|c|c|c|}
\hline Tumor type & Identifier & Intervention & Phase & Sponsors \\
\hline Solid tumors & NCT0I023737 & HCQ + Vorinostat & I & CTRC at UTHSCSA, Merck, NCl \\
\hline Multiple myeloma & NCT00568880 & $\mathrm{HCQ}+$ Bortezomib & $1 / 2$ & U Penn, Millennium \\
\hline Brain, CNS tumors & NCT00486603 & HCQ + Temozolomide/radiation & $1 / 2$ & U Penn, CTEP, NCl \\
\hline Solid tumors & NCT007I4I8I & HCQ + Temozolomide & I & U Penn, Merck \\
\hline Solid tumors & NCT0090983I & $\mathrm{HCQ}+$ Temsirolimus & 1 & U Penn, Pfizer \\
\hline Renal cancer & NCTOI5IOII9 & $\mathrm{HCQ}+\mathrm{RAD00I}$ & $\mathrm{I} / 2$ & U Penn \\
\hline Colorectal cancer & NCT0I 206530 & HCQ + FOLFOX/Bevacizumab & $1 / 2$ & U Penn \\
\hline Pancreatic cancer & NCT0I506973 & $\mathrm{HCQ}+$ Gemcitabine/Abraxane & $\mathrm{I} / 2$ & U Penn \\
\hline Pancreatic cancer & NCTOII 28296 & $\mathrm{HCQ}+$ Gemcitabine & 1 & U Pittsburgh \\
\hline Renal cancer & NCT0II44I69 & $\mathrm{HCQ}$ & 1 & U Pittsburgh \\
\hline Prostate cancer & NCT00726596 & $\mathrm{HCQ}$ & 2 & $\mathrm{CINJ}, \mathrm{NCl}$ \\
\hline Prostate cancer & NCT00786682 & HCQ + Docetaxel & 2 & $\mathrm{CINJ}, \mathrm{NCl}$ \\
\hline Breast cancer & NCT00765765 & HCQ + Ixabepilone & $\mathrm{I} / 2$ & $\mathrm{CINJ}, \mathrm{NCl}$ \\
\hline Lung cancer & NCT00728845 & HCQ + Bevacizumab, Carboplatin, Paclitaxel & $1 / 2$ & $\mathrm{CINJ}, \mathrm{NCl}$ \\
\hline Solid tumors & NCT008I3423 & $\mathrm{HCQ}+$ Sunitinib & 1 & CINJ, CTEP, $\mathrm{NCl}$ \\
\hline Colorectal cancer & NCT0I006369 & HCQ + Capecitabine, Oxaliplatin, Bevacizumab & 2 & U Med and Dent New Jersey, $\mathrm{NCl}$ \\
\hline Solid tumors & NCT0I 266057 & $\mathrm{HCQ}+$ Sirolimus, Vorinostat & 1 & MD Anderson Cancer Center \\
\hline
\end{tabular}

Abbreviations: HCQ, hydroxychloroquine; CTRC at UTHSCSA, Cancer Therapy and Research Center at University of Texas Health Science Center San Antonio; $\mathrm{NCl}$, National Cancer Institute; CINJ, Cancer Institute of New Jersey; CTEP, Cancer Therapy Evaluation Program.

further investigation of lucanthone as a novel autophagy inhibitor with potential clinical applications in combination with anticancer agents that induce this survival pathway.

A second novel inhibitor of autophagy that warrants further investigation as an anticancer agent is Lys05. This new drug shares some structural homology with CQ and was specifically designed to inhibit autophagy more effectively through the inclusion of two aminoquinoline rings, a triamine linker, and C-7 chlorine.$^{69}$ Lys05 is a water-soluble compound that accumulates more readily within the lysosome and has improved deacidification effects compared with HCQ. Similar to lucanthone, Lys05 displayed significantly higher anticancer activity than CQ/HCQ in preclinical models without inducing significant observable toxicity. Additional studies are planned to further investigate the therapeutic anticancer potential of Lys 05 as a novel inhibitor of autophagy.

\section{Conclusion and future directions}

Defining the role(s) of autophagy in malignant pathogenesis and in the regulation of therapeutic sensitivity is an important and challenging endeavor. A plethora of studies conducted to date have clearly shown that the induction of autophagy can be associated with both the execution of cell death as well as the protection from prodeath stimuli including metabolic stress and treatment with anticancer agents. These observations have generated many important scientific questions and opened up new fields of autophagy-related investigation within the context of cancer. Based on the currently available data in the literature, it is highly likely that the cellular consequences of autophagy induction are cell type-specific and treatment-dependent.

Several key issues that need to be better addressed experimentally include elucidating the individual roles of oncogenes in the regulation of cellular autophagy activity, defining the impact of the tumor microenvironment on autophagy function, and determining the most effective approaches to therapeutically target autophagy for cancer therapy. Of particular interest related to the latter issue regarding the modulation of autophagy as an anticancer strategy, numerous studies have demonstrated that the pharmacological and/or genetic disruption of autophagy in combination with an array of anticancer agents or ionizing radiation yields significant therapeutic benefit in preclinical cancer models. These findings have been clinically translated and more than 20 clinical trials investigating the established inhibitors of autophagy CQ and HCQ in combination with a variety of conventional and targeted cancer therapies are currently ongoing. These studies will likely yield important information regarding the safety and preliminary efficacy of these autophagy inhibitors as potential augmenters of cancer therapy.

Given that the ocular toxicities and minimal single agent anticancer efficacy of CQ and HCQ may limit their future applications in cancer therapy, the discovery of novel autophagy inhibitors is a high priority. Lucanthone and Lys 05 are two new and exciting autophagy inhibitors that have demonstrated superior efficacy and tolerability compared with $\mathrm{CQ} / \mathrm{HCQ}$ in preclinical studies. Ongoing preclinical 
studies and planned clinical trials with lucanthone and Lys05 will provide critical information regarding their potential applications in cancer therapy.

\section{Acknowledgments}

This work was supported by funding from The Institute for Drug Development, Cancer Therapy and Research Center at The University of Texas Health Science Center at San Antonio, USA.

\section{Disclosure}

The authors report no conflicts of interest in this work.

\section{References}

1. Carew JS, Nawrocki ST, Cleveland JL. Modulating autophagy for therapeutic benefit. Autophagy. 2007;3(5):464-467.

2. Kuma A, Hatano M, Matsui M, et al. The role of autophagy during the early neonatal starvation period. Nature. 2004;432(7020):1032-1036.

3. Komatsu M, Waguri S, Ueno T, et al. Impairment of starvationinduced and constitutive autophagy in Atg7-deficient mice. $J$ Cell Biol. 2005;169(3):425-434.

4. Jung CH, Jun CB, Ro SH, et al. ULK-Atg13-FIP200 complexes mediate mTOR signaling to the autophagy machinery. Mol Biol Cell. 2009;20(7):1992-2003.

5. Funderburk SF, Wang QJ, Yue Z. The Beclin 1-VPS34 complex - at the crossroads of autophagy and beyond. Trends Cell Biol. 2010;20(6): 355-362.

6. Pankiv S, Clausen TH, Lamark T, et al. p62/SQSTM1 binds directly to Atg8/LC3 to facilitate degradation of ubiquitinated protein aggregates by autophagy. J Biol Chem. 2007;282(33):24131-24145.

7. Schweers RL, Zhang J, Randall MS, et al. NIX is required for programmed mitochondrial clearance during reticulocyte maturation. Proc Natl Acad Sci U S A. 2007;104(49):19500-19505.

8. Loewith R, Jacinto E, Wullschleger S, et al. Two TOR complexes, only one of which is rapamycin sensitive, have distinct roles in cell growth control. Mol Cell. 2002;10(3):457-468.

9. Hara K, Maruki Y, Long X, et al. Raptor, a binding partner of target of rapamycin (TOR), mediates TOR action. Cell. 2002;110(2):177-189.

10. Kim DH, Sarbassov DD, Ali SM, et al. mTOR interacts with raptor to form a nutrient-sensitive complex that signals to the cell growth machinery. Cell. 2002;110(2):163-175.

11. Peterson TR, Laplante M, Thoreen CC, et al. DEPTOR is an mTOR inhibitor frequently overexpressed in multiple myeloma cells and required for their survival. Cell. 2009;137(5):873-886.

12. Sancak Y, Thoreen CC, Peterson TR, et al. PRAS40 is an insulinregulated inhibitor of the mTORC1 protein kinase. Mol Cell. 2007;25(6):903-915.

13. Vander Haar E, Lee SI, Bandhakavi S, Griffin TJ, Kim DH. Insulin signalling to mTOR mediated by the Akt/PKB substrate PRAS40. Nat Cell Biol. 2007;9(3):316-323.

14. Neshat MS, Mellinghoff IK, Tran C, et al. Enhanced sensitivity of PTEN-deficient tumors to inhibition of FRAP/mTOR. Proc Natl Acad Sci U S A. 2001;98(18):10314-10319.

15. Podsypanina K, Lee RT, Politis $\mathrm{C}$, et al. An inhibitor of mTOR reduces neoplasia and normalizes p70/S6 kinase activity in Pten $+/-$ mice. Proc Natl Acad Sci U S A. 2001;98(18):10320-10325.

16. Kamada Y, Funakoshi T, Shintani T, Nagano K, Ohsumi M, Ohsumi Y. Tor-mediated induction of autophagy via an Apg1 protein kinase complex. J Cell Biol. 2000;150(6):1507-1513.

17. Yang Z, Klionsky DJ. Mammalian autophagy: core molecular machinery and signaling regulation. Curr Opin Cell Biol. 2010;22(2):124-131.
18. He C, Klionsky DJ. Regulation mechanisms and signaling pathways of autophagy. Аппи Rev Genet. 2009;43:67-93.

19. Sun SY, Rosenberg LM, Wang X, et al. Activation of Akt and eIF4E survival pathways by rapamycin-mediated mammalian target of rapamycin inhibition. Cancer Res. 2005;65(16):7052-7058.

20. Easton JB, Houghton PJ. mTOR and cancer therapy. Oncogene. 2006;25(48):6436-6446.

21. Oberstein A, Jeffrey PD, Shi Y. Crystal structure of the Bcl-XL-Beclin 1 peptide complex: Beclin 1 is a novel BH3-only protein.J Biol Chem. 2007; 282(17):13123-13132.

22. Zalckvar E, Berissi H, Mizrachy L, et al. DAP-kinase-mediated phosphorylation on the $\mathrm{BH} 3$ domain of beclin 1 promotes dissociation of beclin 1 from Bcl-XL and induction of autophagy. EMBO Rep. 2009;10(3):285-292.

23. Wei Y, Pattingre S, Sinha S, Bassik M, Levine B. JNK1-mediated phosphorylation of Bcl-2 regulates starvation-induced autophagy. Mol Cell. 2008;30(6):678-688.

24. Shi CS, Kehrl JH. TRAF6 and A20 regulate lysine 63-linked ubiquitination of Beclin-1 to control TLR4-induced autophagy. Sci Signal. 2010;3(123):ra42.

25. Yue Z, Jin S, Yang C, Levine AJ, Heintz N. Beclin 1, an autophagy gene essential for early embryonic development, is a haploinsufficient tumor suppressor. Proc Natl Acad Sci U S A. 2003;100(25): 15077-15082.

26. Qu X, Yu J, Bhagat $\mathrm{G}$, et al. Promotion of tumorigenesis by heterozygous disruption of the beclin 1 autophagy gene. J Clin Invest. 2003;112(12): 1809-1820.

27. Crighton D, Wilkinson S, O'Prey J, et al. DRAM, a p53-induced modulator of autophagy, is critical for apoptosis. Cell. 2006;126(1): 121-134.

28. Crighton D, Wilkinson S, Ryan KM. DRAM links autophagy to p53 and programmed cell death. Autophagy. 2007;3(1):72-74.

29. Crighton D, O'Prey J, Bell HS, Ryan KM. p73 regulates DRAMindependent autophagy that does not contribute to programmed cell death. Cell Death Differ. 2007;14(6):1071-1079.

30. Yee KS, Wilkinson S, James J, Ryan KM, Vousden KH. PUMA- and Bax-induced autophagy contributes to apoptosis. Cell Death Differ. 2009;16(8):1135-1145

31. Feng Z, Hu W, de Stanchina E, et al. The regulation of AMPK beta1, TSC2, and PTEN expression by p53: stress, cell and tissue specificity, and the role of these gene products in modulating the IGF-1-AKTmTOR pathways. Cancer Res. 2007;67(7):3043-3053.

32. Budanov AV, Karin M. p53 target genes sestrin 1 and sestrin 2 connect genotoxic stress and mTOR signaling. Cell. 2008;134(3):451-460.

33. O'Prey J, Skommer J, Wilkinson S, Ryan KM. Analysis of DRAMrelated proteins reveals evolutionarily conserved and divergent roles in the control of autophagy. Cell Cycle. 2009;8(14):2260-2265.

34. Mah LY, O'Prey J, Baudot AD, Hoekstra A, Ryan KM. DRAM-1 encodes multiple isoforms that regulate autophagy. Autophagy. 2012;8(1):18-28.

35. Mathew R, Karp CM, Beaudoin B, et al. Autophagy suppresses tumorigenesis through elimination of p62. Cell. 2009;137(6):1062-1075.

36. Cadwell K, Liu JY, Brown SL, et al. A key role for autophagy and the autophagy gene Atg1611 in mouse and human intestinal Paneth cells. Nature. 2008;456(7219):259-263.

37. Hara T, Nakamura K, Matsui M, et al. Suppression of basal autophagy in neural cells causes neurodegenerative disease in mice. Nature. 2006;441(7095):885-889.

38. Mathew R, Karantza-Wadsworth V, White E. Role of autophagy in cancer. Nat Rev Cancer. 2007;7(12):961-967.

39. Li BX, Li CY, Peng RQ, et al. The expression of beclin 1 is associated with favorable prognosis in stage IIIB colon cancers. Autophagy. 2009;5(3):303-306.

40. Chen Y, Lu Y, Lu C, Zhang L. Beclin-1 expression is a predictor of clinical outcome in patients with esophageal squamous cell carcinoma and correlated to hypoxia-inducible factor (HIF)-1alpha expression. Pathol Oncol Res. 2009;15(3):487-493. 
41. Pirtoli L, Cevenini G, Tini P, et al. The prognostic role of Beclin 1 protein expression in high-grade gliomas. Autophagy. 2009;5(7):930-936.

42. Ding ZB, Shi YH, Zhou J, et al. Association of autophagy defect with a malignant phenotype and poor prognosis of hepatocellular carcinoma. Cancer Res. 2008;68(22):9167-9175.

43. Wan XB, Fan XJ, Chen MY, et al. Elevated Beclin 1 expression is correlated with HIF-1alpha in predicting poor prognosis of nasopharyngeal carcinoma. Autophagy. 2010;6(3):395-404.

44. Lazova R, Klump V, Pawelek J. Autophagy in cutaneous malignant melanoma. J Cutan Pathol. 2010;37(2):256-268.

45. Yoshioka A, Miyata H, Doki Y, et al. LC3, an autophagosome marker, is highly expressed in gastrointestinal cancers. Int J Oncol. 2008;33(3):461-468.

46. Kondo Y, Kanzawa T, Sawaya R, Kondo S. The role of autophagy in cancer development and response to therapy. Nat Rev Cancer. 2005;5(9): 726-734.

47. Lum JJ, Bauer DE, Kong M, et al. Growth factor regulation of autophagy and cell survival in the absence of apoptosis. Cell. 2005;120(2):237-248.

48. Bellot G, Garcia-Medina R, Gounon P, et al. Hypoxia-induced autophagy is mediated through hypoxia-inducible factor induction of BNIP3 and BNIP3L via their BH3 domains. Mol Cell Biol. 2009;29(10): 2570-2581.

49. Band M, Joel A, Hernandez A, Avivi A. Hypoxia-induced BNIP3 expression and mitophagy: in vivo comparison of the rat and the hypoxia-tolerant mole rat, Spalax ehrenbergi. FASEB J. 2009;23(7): 2327-2335

50. DeYoung MP, Horak P, Sofer A, Sgroi D, Ellisen LW. Hypoxia regulates TSC1/2-mTOR signaling and tumor suppression through REDD1mediated 14-3-3 shuttling. Genes Dev. 2008;22(2):239-251.

51. Glick D, Barth S, Macleod KF. Autophagy: cellular and molecular mechanisms. J Pathol. 2010;221(1):3-12.

52. Hippert MM, O’Toole PS, Thorburn A. Autophagy in cancer: good, bad, or both? Cancer Res. 2006;66(19):9349-9351.

53. Kreuzaler P, Watson CJ. Killing a cancer: what are the alternatives? Nat Rev Cancer. 2012;12(6):411-424.

54. Kroemer G, Levine B. Autophagic cell death: the story of a misnomer. Nat Rev Mol Cell Biol. 2008;9(12):1004-1010.

55. Degenhardt K, Mathew R, Beaudoin B, et al. Autophagy promotes tumor cell survival and restricts necrosis, inflammation, and tumorigenesis. Cancer Cell. 2006;10(1):51-64.

56. Amaravadi RK, Yu D, Lum JJ, et al. Autophagy inhibition enhances therapy-induced apoptosis in a Myc-induced model of lymphoma. J Clin Invest. 2007;117(2):326-336.

57. Bellodi C, Lidonnici MR, Hamilton A, et al. Targeting autophagy potentiates tyrosine kinase inhibitor-induced cell death in Philadelphia chromosome-positive cells, including primary CML stem cells. J Clin Invest. 2009;119(5):1109-1123.

58. Carew JS, Espitia CM, Esquivel JA 2nd, et al. Lucanthone is a novel inhibitor of autophagy that induces cathepsin D-mediated apoptosis. J Biol Chem. 2011;286(8):6602-6613.

59. Carew JS, Medina EC, Esquivel JA 2nd, et al. Autophagy inhibition enhances vorinostat-induced apoptosis via ubiquitinated protein accumulation. J Cell Mol Med. 2010;14(10):2448-2459.

60. Carew JS, Nawrocki ST, Giles FJ, Cleveland JL. Targeting autophagy: a novel anticancer strategy with therapeutic implications for imatinib resistance. Biologics. 2008;2(2):201-204.

61. Takeuchi H, Kondo Y, Fujiwara K, et al. Synergistic augmentation of rapamycin-induced autophagy in malignant glioma cells by phosphatidylinositol 3-kinase/protein kinase B inhibitors. Cancer Res. 2005;65(8):3336-3346.

62. Zhu K, Dunner K Jr, McConkey DJ. Proteasome inhibitors activate autophagy as a cytoprotective response in human prostate cancer cells. Oncogene. 2010;29(3):451-462.

63. Carew JS, Nawrocki ST, Kahue CN, et al. Targeting autophagy augments the anticancer activity of the histone deacetylase inhibitor SAHA to overcome Bcr-Abl-mediated drug resistance. Blood. 2007;110(1):313-322.
64. Amaravadi RK, Lippincott-Schwartz J, Yin XM, et al. Principles and current strategies for targeting autophagy for cancer treatment. Clin Cancer Res. 2011;17(4):654-666.

65. Glaumann H, Ahlberg J. Comparison of different autophagic vacuoles with regard to ultrastructure, enzymatic composition, and degradation capacity - formation of crinosomes. Exp Mol Pathol. 1987;47(3):346-362.

66. Dassonneville L, Bailly C. Stimulation of topoisomerase II-mediated DNA cleavage by an indazole analogue of lucanthone. Biochem Pharmacol. 1999;58(8):1307-1312.

67. Luo M, Kelley MR. Inhibition of the human apurinic/apyrimidinic endonuclease (APE1) repair activity and sensitization of breast cancer cells to DNA alkylating agents with lucanthone. Anticancer Res. 2004;24(4):2127-2134.

68. Milligan AJ, Katz HR, Leeper DB. Effect of lucanthone hydrochloride on the radiation response of intestine and bone marrow of the Chinese hamster. J Natl Cancer Inst. 1978;60(5):1023-1028.

69. McAfee Q, Zhang Z, Samanta A, et al. Autophagy inhibitor Lys05 has single-agent antitumor activity and reproduces the phenotype of a genetic autophagy deficiency. Proc Natl Acad Sci USA. 2012;109(21): 8253-8258.

70. Yazbeck VY, Buglio D, Georgakis GV, et al. Temsirolimus downregulates p21 without altering cyclin D1 expression and induces autophagy and synergizes with vorinostat in mantle cell lymphoma. Exp Hematol. 2008;36(4):443-450.

71. Cao C, Subhawong T, Albert JM, et al. Inhibition of mammalian target of rapamycin or apoptotic pathway induces autophagy and radiosensitizes PTEN null prostate cancer cells. Cancer Res. 2006;66(20):10040-10047.

72. Alonso MM, Jiang H, Yokoyama T, et al. Delta-24-RGD in combination with RAD001 induces enhanced anti-glioma effect via autophagic cell death. Mol Ther. 2008;16(3):487-493.

73. Shao Y, Gao Z, Marks PA, Jiang X. Apoptotic and autophagic cell death induced by histone deacetylase inhibitors. Proc Natl Acad Sci U S A. 2004;101(52):18030-18035.

74. Ellis L, Bots M, Lindemann RK, et al. The histone deacetylase inhibitors LAQ824 and LBH589 do not require death receptor signaling or a functional apoptosome to mediate tumor cell death or therapeutic efficacy. Blood. 2009;114(2):380-393.

75. Ding WX, Ni HM, Gao W, et al. Oncogenic transformation confers a selective susceptibility to the combined suppression of the proteasome and autophagy. Mol Cancer Ther. 2009;8(7):2036-2045.

76. Basciani S, Vona R, Matarrese P, et al. Imatinib interferes with survival of multi drug resistant Kaposi's sarcoma cells. FEBS Lett. 2007;581(30):5897-5903

77. Bilir A, Erguven M, Oktem G, et al. Potentiation of cytotoxicity by combination of imatinib and chlorimipramine in glioma. Int $J$ Oncol. 2008;32(4):829-839.

78. Ertmer A, Huber V, Gilch S, et al. The anticancer drug imatinib induces cellular autophagy. Leukemia. 2007;21(5):936-942.

79. Milano V, Piao Y, LaFortune T, de Groot J. Dasatinib-induced autophagy is enhanced in combination with temozolomide in glioma. Mol Cancer Ther. 2009;8(2):394-406.

80. Martin AP, Park MA, Mitchell C, et al. BCL-2 family inhibitors enhance histone deacetylase inhibitor and sorafenib lethality via autophagy and overcome blockade of the extrinsic pathway to facilitate killing. Mol Pharmacol. 2009;76(2):327-341.

81. Huang HL, Chen YC, Huang YC, et al. Lapatinib induces autophagy, apoptosis and megakaryocytic differentiation in chronic myelogenous leukemia K562 cells. PLoS One. 2011;6(12):e29014.

82. Bursch W, Ellinger A, Kienzl H, et al. Active cell death induced by the anti-estrogens tamoxifen and ICI 164384 in human mammary carcinoma cells (MCF-7) in culture: the role of autophagy. Carcinogenesis. 1996;17(8):1595-1607.

83. Turzanski J, Daniels I, Haynes AP. Involvement of macroautophagy in the caspase-independent killing of Burkitt lymphoma cell lines by rituximab. Br J Haematol. 2009;145(1):137-140. 
84. Giannopoulou E, Antonacopoulou A, Matsouka P, Kalofonos HP. Autophagy: novel action of panitumumab in colon cancer. Anticancer Res. 2009;29(12):5077-5082.

85. Li X, Fan Z. The epidermal growth factor receptor antibody cetuximab induces autophagy in cancer cells by downregulating HIF-1alpha and Bcl-2 and activating the beclin $1 / \mathrm{hVps} 34$ complex. Cancer Res. 2010;70(14):5942-5952.
86. Voss V, Senft C, Lang V, et al. The pan-Bcl-2 inhibitor (-)-gossypol triggers autophagic cell death in malignant glioma. Mol Cancer Res. 2010;8(7):1002-1016.

87. Maiuri MC, Criollo A, Tasdemir E, et al. BH3-only proteins and BH3 mimetics induce autophagy by competitively disrupting the interaction between Beclin 1 and $\mathrm{Bcl}-2 / \mathrm{Bcl}-\mathrm{X}(\mathrm{L})$. Autophagy. 2007;3(4):374-376.

\section{Publish your work in this journal}

Cancer Management and Research is an international, peer-reviewed open access journal focusing on cancer research and the optimal use of preventative and integrated treatment interventions to achieve improved outcomes, enhanced survival and quality of life for the cancer patient The journal welcomes original research, clinical \& epidemiological studies, reviews \& evaluations, guidelines, expert opinion \& commentary, case reports \& extended reports. The manuscript management system is completely online and includes a very quick and fair peerreview system, which is all easy to use. Visit http://www.dovepress.com/ testimonials.php to read real quotes from published authors.

Submit your manuscript here: http://www.dovepress.com/cancer-management-and-research-journal 